Published in final edited form as:

Infect Control Hosp Epidemiol. 2015 January ; 36(1): 2-13. doi:10.1017/ice.2014.1.

\title{
Cost-Benefit Analysis from the Hospital Perspective of Universal Active Screening Followed by Contact Precautions for Methicillin-resistant Staphylococcus aureus Carriers
}

\author{
James A. McKinnell, MD ${ }^{1,2}$, Sarah M. Bartsch, MPH ${ }^{3,4}$, Bruce Y. Lee, MD, MBA ${ }^{3}$, Susan S. \\ Huang, MD, $\mathrm{MPH}^{5}$, and Loren G. Miller, MD, MPH${ }^{1}$ \\ ${ }^{1}$ Infectious Disease Clinical Outcomes Research Unit (ID-CORE), Division of Infectious Disease, \\ Los Angeles Biomedical Research Institute, Harbor-UCLA Medical Center, Torrance, CA \\ ${ }^{2}$ Torrance Memorial Medical Center, Torrance, CA \\ ${ }^{3}$ Public Health Computational and Operations Research (PHICOR) Group, Johns Hopkins \\ Bloomberg School of Public Health, Baltimore, MD \\ ${ }^{4}$ Department of Industrial Engineering, University of Pittsburgh, Pittsburgh, PA \\ ${ }^{5}$ Division of Infectious Diseases and Health Policy Research Institute, University of California, \\ Irvine School of Medicine, Irvine, California
}

\begin{abstract}
Objective-There are data suggesting potential benefit to screening hospitalized patients for MRSA colonization followed by contact precautions for carriers. However, MRSA screening, like most prevention interventions, has associated costs that must be put into context of potential benefits. We therefore sought to explore the economic impact to a hospital of universal MRSA screening.
\end{abstract}

Methods-We used a decision tree model to estimate the direct economic impact to an individual hospital of starting universal MRSA screening and contact precautions. Projected costs and benefits were based on literature derived data. Our model examined outcomes of several strategies including non-nares MRSA screening and comparison of culture versus PCR based screening.

Results-Under baseline conditions, the costs of universal MRSA screening and contact precautions outweighed the projected benefits generated by preventing MRSA related infections, resulting in economic costs of $\$ 104,000$ per 10,000 admissions ([ $95 \%$ credibility range, $\$ 83,000$ to $\$ 126,000])$. Cost-savings only occurred when the model used estimates at the extremes of our key parameters. Non-nares screening and PCR-based testing, both of which identified more MRSA colonized persons, resulted in more MRSA infections averted, but increased economic costs of the screening program.

Conclusions-We found that universal MRSA screening, while providing potential benefit in preventing MRSA infection, is relatively costly may be economically burdensome for a hospital. Policy makers should consider the economic burden of MRSA screening and contact precautions in relation to other interventions when choosing programs to improve patient safety and outcomes. 
Methicillin-resistant Staphylococcus aureus (MRSA) is a major cause of healthcareassociated infections, with particularly high incidence in the United States (U.S.), Asia, and parts of Europe.(1,2) MRSA infections amongst hospitalized patients can result in devastating morbidity and significant mortality. Preventing spread of MRSA amongst hospitalized patients is a priority for hospitals, public health officials, and policy makers.

Amongst hospital-based strategies to prevent MRSA infections, MRSA screening followed by subsequent contact precautions is a common strategy used by U.S. hospitals.(3-5) MRSA screening and contact precautions in populations with high MRSA prevalence has demonstrated effectiveness in reducing transmission and newly acquired infections.(6-12) Guidelines from the Society for Healthcare Epidemiology of America (SHEA) recommend active surveillance for MRSA,(5) but this recommendation has been challenged and is not universally adopted as a gold standard across the US.(13) Public concern about MRSA infections has led to a number of U.S. state legislatures passing laws mandating that hospitals perform active surveillance for MRSA in selected populations, particularly for intensive care units (ICUs), regardless of underlying MRSA prevalence.(14) Others have advocated for even broader, hospital-wide programs of universal surveillance and isolation. $(15,16)$ Support for broader screening has come from investigators suggesting that hospitalwide, universal surveillance may be a cost-effective strategy when considered from a societal perspective. $(16,17)$

While universal surveillance may be cost-effective from a societal perspective, hospitals considering implementation of universal surveillance must consider the additional costs incurred from surveillance and isolation, and contact precautions that are not reimbursed. The economic impact to an individual hospital may represent an important barriers to implementation. To examine the potential economic barriers to implementation of universal MRSA surveillance, we developed a decision tree model to quantify the costs and benefits of implementing universal MRSA surveillance in a hospital. We believe that results of our model may help hospitals, state, and national policymakers understand the economic impact of universal surveillance on a hospital.

\section{Methods}

We developed a decision tree model to estimate the economic impact of adopting a hospitalwide, universal active surveillance program for MRSA with subsequent contact precautions for all MRSA carriers. Specifically, we compared the costs of the surveillance and contact precaution program against the projected economic benefits of preventing secondary MRSA infections. The cost-benefit analysis was conducted from the individual hospital perspective and compared against no screening. We report results for 10,000 inpatient admissions, which can readily be converted to any number of admissions (e.g., a 500 admission hospital would divide 10,000 by 20 ). Based on a recently completed systematic literature review, our baseline conditions assume an MRSA nares colonization prevalence of $7.3 \%$ in US hospitals.(18) Based on the same review, our baseline conditions assume a ratio of nasal carriage to total body MRSA colonization.(18) 


\section{Development of the Decision Analysis Model}

We used TreeAgo Pro 2013 (Williamstown, MA) to build a cost-benefit model to examine the implementation of a hospital wide, universal MRSA screening program from the hospital perspective (Figure 1, Appendix 1). Briefly, each patient entering the model was categorized as either MRSA colonized or not colonized. Each patient was defined as carrying MRSA at each pre-specified body site, based on our previous systematic review of the literature.(18, 19) Input parameters for costs, MRSA epidemiology, MRSA testing characteristics, and potential benefits of contact precautions were extracted from the literature and summarized in Table 1 . Several key parameters were the focus of additional sensitivity analysis as described below.

All patients underwent MRSA screening on admission using one of the following screening strategies: 1) nares only, 2) nares and oropharynx, or 3) multi-site swabbing. In our model, patients test either positive or negative for MRSA depending on their colonization status and the sensitivity and specificity of the test (Table 1). In our model, patients testing positive, regardless of their true colonization status (i.e., both true and false positives) were placed in contact precautions for the remainder of their hospitalization. (Contact precautions were not used pre-emptively pending test results.) Separate analyses were conducted to compare screening with chromagar methods and PCR-based methods. We used each of these models to test the hypothesis that better screening, either by swabbing more body sites or using more sensitive diagnostic testing, would improve the benefit of screening and contact precautions.

\section{Determination of Estimated Costs}

The costs of the screening and contact precaution program are based on the material and labor costs to the hospital of both the screening procedures and the contact precautions (Table 1). For each simulation, the cost for each screening strategy was calculated as:

\section{(Cost of Contact Precautions + Cost of Screening)}

The screening costs included the cost of swab(s), which assumed each body site was tested with its own swab. The costs of routine culture were based on chromogenic agar plates where each patient required one plate. For multiple body site testing, we allowed for split plate testing, maintaining one chromogenic agar plate per patient. For PCR based testing, we included the cost of PCR materials. For multisite PCR testing, we assumed that the multiple body site samples could be batched for one PCR test. For both culture and PCR testing, we included the cost of technicians' time to process the sample.

The cost of contact precautions included the material costs of additional gloves and gowns used and additional nursing time to don and doff gloves and gowns before each entry into patient rooms for the duration of their hospital stay. The cost of nursing time was estimated from the average hourly nurse wage from the U.S. Bureau of Labor and Statistics (national statistics for 2012).(20) We did not include additional support for infection control personnel to manage the program, nor did we include start-up costs, e.g. staff training. Costs were converted from previous years to 2014 U.S. dollars using a 3\% discount rate. 


\section{Determination of Projected Benefits}

The potential benefit to a hospital of a screening and contact precaution program is averting secondary MRSA infections through the appropriate implementation of contact precautions. The cost of an MRSA infection to the hospital was calculated from the MRSA attributable length of stay and the cost of a hospital bed day, following a method described by Graves. (21) These additional days represents the opportunity cost of a lost bed day, where a bed could have been used by another patient to generate revenue.

For each simulation, the benefit for each screening strategy was calculated as:

(Number Infections Averted $\times$ MRSA Attributable Length of Stay $\times$ Cost of Lost Bed Day)

\section{Cost-Benefit Analysis}

The economic impact of adopting a universal surveillance and contact precautions program was based on the difference between the benefits (i.e., cost-savings from averting MRSA infections) and intervention costs. For each simulation, the economic impact to the hospital for each screening strategy was calculated as:

(Number Infections Averted $\times$ MRSA Attributable Length of Stay $\times$ Cost of Lost Bed Day $)-($ Cost of Contact Precautions + Cost of Screening)

The optimal strategy was defined as the strategy with the best cost-benefit to the hospital; i.e. cost-neutral (costs $=$ benefit) or cost-saving (cost<benefit).

\section{Model Simulations}

Each simulation sent 1,000 hospital admissions through the model 1,000 times for a total of 1 million trials. Our model results are expressed as outcomes per 10,000 hospital admissions and include: total number of colonized patients identified by the surveillance program, total number of patients placed in contact precautions, number of secondary infections averted by the intervention, and the cost-benefit (costs averted by preventing MRSA infections intervention costs) of the intervention. Probabilistic sensitivity analyses (i.e., Monte Carlo simulations) were performed for each simulation, varying each parameter throughout their ranges (Table 1). The distributions varied in the Monte Carlo simulations account for variability in known parameters and were created based on the available data (e.g., variability across studies or ranges/confidence intervals from one source).

\section{Sensitivity Analyses of Key Model Estimates}

Additional one- and two-way sensitivity analysis varied key parameters in the model to determine their impact on the projected cost-benefit of each strategy. These parameters include number of MRSA infections averted per isolated MRSA carrier, MRSA attributable length of say, and MRSA prevalence among admitted patients. Our baseline conditions assumed 0.005 infections averted per isolated MRSA carrier, a 7.3\% nares colonization prevalence, and a 6-day attributable length of stay.

We estimated the number of MRSA infections averted per MRSA carrier placed in contact precautions as 0.005 , based on currently available estimates from the literature. $(8-11,16)$ 
However, we recognize that the literature on MRSA contact precautions efficacy is mixed and based largely on select populations or higher prevalence settings.(22) We therefore used data from a randomized trial of hospital-wide, universal screening and contact precautions combined with chlorhexidine bathing as an extreme estimate for the efficacy of universal active screening followed by contact precautions, i.e. 0.05 MRSA cases per patient placed in contact precautions and decolonized.(23) Using this data, we varied contact precaution efficacy in reducing MRSA infection from 0.005 (base-case estimate), to 0.015 (optimistic estimate), to 0.03 (extreme estimate) MRSA cases averted per MRSA carrier placed in contact precautions.

We estimated the additional hospital length of stay attributed to MRSA was 6 additional days. However, as the attributable length of stay due to an MRSA depends on the type of acquired infection (e.g., skin and soft tissue infection vs. bacteremia vs. endocarditis), we ranged this from 2 to 16 additional hospital days (based on estimates of attributable length of stay for different types of infection available from the literature(24-28)).

We designed our base-case scenario with MRSA nares colonization prevalence of $7.3 \%$ and the total body MRSA colonization prevalence was $9.5 \%$ based on a recent systematic literature review on MRSA colonization.(18) Based on this review, we varied total body MRSA colonization to range from $9.5 \%$ to $30 \%$ and nares colonization ranging from $7.3 \%$ to $22 \%$ to determine if hospitals with higher burden of MRSA may benefit from universal active surveillance.

\section{Results}

Table 2 shows results for our baseline conditions for both chromagar and PCR methods. A program of nares screening for all patients admitted to a hospital using chromogenic agar identified 545 MRSA colonized patients/10,000 admissions (95\% credibility range, 420-683 colonized patients/10,000 admissions) and averted 3 cases of invasive MRSA disease (95\% credibility range 2-3.4), preventing 18 additional hospitalization days and saving $\$ 24,740$ (95\% credibility range, $18,920-30,960)$ (Table 2 ). Nares surveillance program improperly placed 79 patients in contact precautions. The overall economic impact to the hospital was a $\$ 237,494$ loss (95\% credibility range, $\$ 199,867$ to $\$ 283,610$ ) for 10,000 admissions (Table 2).

Table 3 shows results assuming an extreme estimate of contact precaution efficacy $(0.03$ infections averted per carrier isolated). Results were similar to less efficacious scenarios, resulting in economic losses over $\$ 104,000$. Our results can be used to estimate the associated costs and cost-benefit for a hospital with any number of admission. For example, a hospital with 2,500 admission implementing nares only screening (under the baseline conditions) would isolate 156 patients $(625 / 4)$ resulting in losses of $\$ 56,874(\$ 227,494 / 4)$.

\section{Multi-site testing for MRSA}

Under the baseline conditions, both the nares/oropharynx and three-site surveillance improved the number of correctly identified carriers and improved the number of infections avoided. The multi-site swabbing approaches resulted in modest increases in prevention of 
infection but resulted in more substantial economic losses to the hospital (Tables 2 and 3). Multi-site screening was cost minimizing with relatively high estimates of attributable length of stay for MRSA infection (10 days) and optimistic estimates of contact precaution efficacy (0.03 infections per MRSA carrier isolated). Nares alone or nares/oropharynx screening were never cost-minimizing when MRSA colonization prevalence was $₫ 30 \%$ (Figure 2).

\section{PCR versus chromatogenic agar}

Under the baseline conditions, PCR-based surveillance improved the number of correctly identified carriers and improved the number of infections avoided (Tables 2 and 3). PCRbased nares surveillance resulted in modest improvements in infection prevention, but resulted in an economic loss of $\$ 546,583$ (95\% credibility range, $\$ 493,459$ to $\$ 599,579$ ) for 10,000 admissions (Table 2). PCR screening was never cost-minimizing under any of the conditions tested.

\section{Sensitivity Analyses}

Figure 2 displays two-way sensitivity analyses for the MRSA attributable length of stay (2-16 days) and number of infections averted (0.005-0.03 per carrier placed in contact precautions) by MRSA admission prevalence $(9.5 \%-30 \%)$. The graphs display the screening strategy that results in the best cost-benefit to the hospital (i.e., optimal strategy). Under most model assumptions, no screening resulted in the best cost-benefit to the hospital. Under baseline conditions, the only time that universal screening was cost-saving occurred with extreme estimates for contact precaution efficacy ( 0.03 cases averted per patient placed in contact precautions) and when MRSA attributable length of stay was $>10$. With MRSA admission prevalence of $30 \%$, universal screening became cost-saving was with extreme contact precaution efficacy ( 0.03 cases averted per MRSA carrier placed in contact precautions) and when MRSA attributable length of stay was $>8$ days.

\section{Discussion}

We found that even though MRSA screening and contact precaution programs may prevent MRSA infections, these programs have substantial cost ( $\$ 103,000)$ per 10,000 admissions. These results demonstrate that there may be substantial economic cost to the adoption of an expanded MRSA surveillance and contact precautions program to include hospital-wide, universal surveillance. Our findings were robust under a wide range of MRSA prevalence, contract precaution efficacy, and attributable length of stay values. Universal active screening followed by contact precautions was never cost-saving even with the number of sites and type of screening was modified. Even using extreme estimates for contact precaution efficacy ( 0.03 infections averted per carrier isolated) and prolonged attributable length of stay for acquired MRSA infections (up to 16 days), universal surveillance would likely incur significant costs for hospitals, even if only nares surveillance was adopted. Our results are for general benchmarking and hospitals of any size can estimate the impact to their own facility based on their own local epidemiology. Thus our results can help inform various decision makers (i.e., infection control specialists, hospital administrators, and even 
insurance companies) about the cost-benefits and how much they can invest in MRSA prevention and control.

Our results are important in the context of understanding the economic barriers to dissemination of potentially effective infection control practices across U.S. hospitals. Lee et al demonstrated that universal MRSA surveillance is cost effective for a wide range of MRSA prevalence and reproductive rates.(16) However, the investigation by Lee(16) and a similar investigation by Hubben and colleagues(17) analyzed the cost-effectiveness of universal surveillance from the societal or third-party perspective. While universal MRSA screening may be beneficial to society (as previously described $(16,17)$ ), our analysis demonstrates that the expanded screening program is economically burdensome for a hospital and that the benefits of screening may only be reaped by later or external beneficiaries (e.g., other hospitals or non-hospital-based care entities). To achieve the population benefits of improved patient quality and improved patient safety for the strategy of universal surveillance and contact precautions, reimbursement to the hospital and financial incentives may need to be significantly changed in order to overcome the economic barriers to dissemination.

We note that the results of our analysis were sensitive to the impact of MRSA invasive disease. Therefore, while a program of hospital-wide, universal surveillance may not be economically feasible, there are likely sub-populations for which screening and contact precautions may result in significant savings for the hospital. For example, the impact of MRSA infection on burn patients or patients following cardiothoracic surgery is substantial and may surpass the range of attributable length of stay analyzed in our investigation. Within these specific high-risk populations, invasive MRSA infection can have disastrous and costly implications. Similarly, the impact of invasive MRSA disease in intensive care units can be substantial. Therefore, universal screening and contact precautions within highrisk patients and intensive care units may be less economically burdensome for a hospital but, further analysis is required before specific suggestions can be made about specific subpopulations.

Another key observation from our study is that our results were sensitive to the efficacy of the MRSA intervention. Although screening and contact precautions may not result in sufficient benefit to overcome the costs of the intervention, more efficacious strategies that result in greater projected benefits may be cost-neutral or even cost-saving. For example, recent investigations of chlorhexidine and mupirocin based MRSA decolonization have shown efficacy $(29,30)$ and may not face such a large economic barrier to implementation. One of the benefits of our methodology is that our model estimates efficacy of the intervention based on the number of infections prevented by application of the intervention. The number of infection prevented per intervention is mathematically related to the number needed to treat and can be calculated for other interventions, such as MRSA decolonization. The cost and benefits of universal screening and contact precautions should be weighed against other infection control strategies, such as decolonization, and other quality improvement initiatives. More work is needed to understand the relative benefits and costs of infection prevention strategies with respect to one another in reducing MRSA or other infections. 
There are limitations to our analysis. Importantly, our analysis was limited by the data we used in our model to establish MRSA contact precaution efficacy in terms of the number of infections prevented. Our model did not specifically analyze pre-emptive isolation strategies. However, our results remained robust, even when using extreme estimates for contact precaution efficacy that far exceed estimates for potential benefits of pre-emptive isolation.(8) Our model did not model potential unintended costs of contact precautions, including falls or reduced patient contact.(31) Including these impacts would have made screening more economically burdensome. That said, there conflicting estimates for the unintended impact of contact precautions,(9) so lack of modeling this consequence may be appropriate. We did not include physician time to don/doff contact precautions (although there visits are counted) as we assumed independent medical staff reimbursed by physician billing. Inclusion of physician time to don/doff contact precautions may be appropriate for some systems, i.e. employed hospitalists would see larger Lastly, our model did not attempt to measure the cumulative impact of universal surveillance and contact precautions. It is likely that as population benefit increases (MRSA declines), the cost-benefit of the program would continue to degrade.

Overall, our results estimate the economic burden that expanded MRSA surveillance and contact precautions will create for a hospital. If health policy experts, legislators, or patient safety advocates hope to implement universal MRSA surveillance on a large scale, they must be aware of the economic barriers to implementation and the potential economic impact from these programs. Without significant improvement in financial reimbursement to hospitals, it seems unlikely that hospital administrators can responsibly adopt expanded MRSA surveillance for the entire hospital population. Finally, many costly interventions may improve patient outcomes, but given money is a limited resource, policy makers should consider our findings in light of other hospital-based interventions to prevent patient harm.

\section{Supplementary Material}

Refer to Web version on PubMed Central for supplementary material.

\section{Acknowledgments}

JM received support from the NIH/NCRR/NCATS UCLA CTSI Grant Number KL2TR000122. JM, SB, BL, SH, and LM received support from the AHRQ Grant Number RC4AI092327. SH has disclosed that she is conducting a clinical trial for which participating hospitals receive contributed product from Sage Products and Molnlycke. No other authors have any conflicts of interest to disclose in relation to this manuscript.

\section{References}

1. Hidron AI, Edwards JR, Patel J, Horan TC, Sievert DM, Pollock DA, et al. NHSN annual update: antimicrobial-resistant pathogens associated with healthcare-associated infections: annual summary of data reported to the National Healthcare Safety Network at the Centers for Disease Control and Prevention, 2006-2007. Infect Control Hosp Epidemiol. Nov; 2008 29(11):996-1011. PubMed PMID: 18947320. Epub 2008/10/25. eng. [PubMed: 18947320]

2. Sader HS, Streit JM, Fritsche TR, Jones RN. Antimicrobial susceptibility of gram-positive bacteria isolated from European medical centres: results of the Daptomycin Surveillance Programme (2002-2004). Clin Microbiol Infect. Sep; 2006 12(9):844-52. PubMed PMID: 16882289. Epub 2006/08/03. eng. [PubMed: 16882289] 
3. Coia JE, Duckworth GJ, Edwards DI, Farrington M, Fry C, Humphreys H, et al. Guidelines for the control and prevention of meticillin-resistant Staphylococcus aureus (MRSA) in healthcare facilities. J Hosp Infect. May; 2006 63(Suppl 1):S1-44. PubMed PMID: 16581155. Epub 2006/04/04. eng. [PubMed: 16581155]

4. Siegel JD, Rhinehart E, Jackson M, Chiarello L, Health Care Infection Control Practices Advisory C. 2007 Guideline for Isolation Precautions: Preventing Transmission of Infectious Agents in Health Care Settings. Am J Infect Control. Dec; 2007 35(10 Suppl 2):S65-164. PubMed PMID: 18068815. [PubMed: 18068815]

5. Muto CA, Jernigan JA, Ostrowsky BE, Richet HM, Jarvis WR, Boyce JM, et al. SHEA guideline for preventing nosocomial transmission of multidrug-resistant strains of Staphylococcus aureus and enterococcus. Infect Control Hosp Epidemiol. May; 2003 24(5):362-86. PubMed PMID: 12785411. [PubMed: 12785411]

6. Huang SS, Yokoe DS, Hinrichsen VL, Spurchise LS, Datta R, Miroshnik I, et al. Impact of routine intensive care unit surveillance cultures and resultant barrier precautions on hospital-wide methicillin-resistant Staphylococcus aureus bacteremia. Clin Infect Dis. Oct 15; 2006 43(8):971-8. PubMed PMID: 16983607. Epub 2006/09/20. eng. [PubMed: 16983607]

7. Safdar N, Marx J, Meyer NA, Maki DG. Effectiveness of preemptive barrier precautions in controlling nosocomial colonization and infection by methicillin-resistant Staphylococcus aureus in a burn unit. Am J Infect Control. Oct; 2006 34(8):476-83. PubMed PMID: 17015152. Epub 2006/10/04. eng. [PubMed: 17015152]

8. Harbarth S, Masuet-Aumatell C, Schrenzel J, Francois P, Akakpo C, Renzi G, et al. Evaluation of rapid screening and pre-emptive contact isolation for detecting and controlling methicillin-resistant Staphylococcus aureus in critical care: an interventional cohort study. Critical care. Feb.2006 10(1):R25. PubMed PMID: 16469125. Pubmed Central PMCID: 1550853. [PubMed: 16469125]

9. Harris AD, Pineles L, Belton B, Johnson JK, Shardell M, Loeb M, et al. Universal glove and gown use and acquisition of antibiotic-resistant bacteria in the ICU: a randomized trial. JAMA : the journal of the American Medical Association. Oct 16; 2013 310(15):1571-80. PubMed PMID: 24097234.

10. Clancy M, Graepler A, Wilson M, Douglas I, Johnson J, Price CS. Active screening in high-risk units is an effective and cost-avoidant method to reduce the rate of methicillin-resistant Staphylococcus aureus infection in the hospital. Infect Control Hosp Epidemiol. Oct; 2006 27(10): 1009-17. PubMed PMID: 17006806. [PubMed: 17006806]

11. Jernigan JA, Titus MG, Groschel DH, Getchell-White S, Farr BM. Effectiveness of contact isolation during a hospital outbreak of methicillin-resistant Staphylococcus aureus. American journal of epidemiology. Mar 1; 1996 143(5):496-504. PubMed PMID: 8610665. [PubMed: 8610665]

12. Lawes T, Edwards B, Lopez-Lozano JM, Gould I. Trends in Staphylococcus aureus bacteraemia and impacts of infection control practices including universal MRSA admission screening in a hospital in Scotland, 2006-2010: retrospective cohort study and time-series intervention analysis. BMJ open. 2012; 2(3) PubMed PMID: 22685226. Pubmed Central PMCID: 3378947.

13. Diekema DJ, Edmond MB. Look before you leap: active surveillance for multidrug-resistant organisms. Clin Infect Dis. Apr 15; 2007 44(8):1101-7. PubMed PMID: 17366459. [PubMed: 17366459]

14. Association for Professionals in Infection Control and Hospital Epidemiology. Map of enacted MRSA laws 2011 [updated 4/21/113/2/2012]. Available from: http://www.apic.org/Resource_/ TinyMceFileManager/Advocacy-PDFs/Static_map_-_MRSA_revised_4-21-11.gif

15. Peterson LR, Diekema DJ. To screen or not to screen for methicillin-resistant Staphylococcus aureus. J Clin Microbiol. Mar; 2010 48(3):683-9. PubMed PMID: 20071548. Pubmed Central PMCID: 2832433. [PubMed: 20071548]

16. Lee BY, Bailey RR, Smith KJ, Muder RR, Strotmeyer ES, Lewis GJ, et al. Universal methicillinresistant Staphylococcus aureus (MRSA) surveillance for adults at hospital admission: an economic model and analysis. Infect Control Hosp Epidemiol. Jun; 2010 31(6):598-606. PubMed PMID: 20402588. Pubmed Central PMCID: 3385994. [PubMed: 20402588]

17. Hubben G, Bootsma M, Luteijn M, Glynn D, Bishai D, Bonten M, et al. Modelling the costs and effects of selective and universal hospital admission screening for methicillin-resistant 
Staphylococcus aureus. PloS one. 2011; 6(3):e14783. PubMed PMID: 21483492. Pubmed Central PMCID: 3069001. [PubMed: 21483492]

18. McKinnell JA, Huang SS, Eells SJ, Cui E, Miller LG. Quantifying the impact of extranasal testing of body sites for methicillin-resistant Staphylococcus aureus colonization at the time of hospital or intenstive care unit admission. Infection Control and Hospital Epidemiology. 2013; 34(2):161-70. [PubMed: 23295562]

19. McKinnell JA, Miller LG, Eells SJ, Cui E, Huang SS. A systematic literature review and metaanalysis of factors associated with methicillin-resistant Staphylococcus aureus colonization at time of hospital or intensive care unit admission. Infect Control Hosp Epidemiol. Oct; 2013 34(10): 1077-86. PubMed PMID: 24018925. Pubmed Central PMCID: 3883507. [PubMed: 24018925]

20. Statistics BoL. Occupational Employment Statistics: May 2012 National Occupation Employment and Wage Estimtaes. http://www.bls.gov2012 [September 4, 2013]

21. Graves N. Economics and preventing hospital-acquired infection. Emerging Infectious Diseases. 2004; 10(4):561-6. [PubMed: 15200842]

22. Dancer SJ. Considering the introduction of universal MRSA screening. J Hosp Infect. Aug; 2008 69(4):315-20. PubMed PMID: 18602183. [PubMed: 18602183]

23. Robicsek A, Beaumont JL, Paule SM, Hacek DM, Thomson RB Jr. Kaul KL, et al. Universal surveillance for methicillin-resistant Staphylococcus aureus in 3 affiliated hospitals. Annals of internal medicine. Mar 18; 2008 148(6):409-18. PubMed PMID: 18347349. [PubMed: 18347349]

24. Abramson MA, Sexton DJ. Nosocomial methicillin-resistant and methicillin-susceptible Staphylococcus aureus primary bacteremia: at what costs? Infect Control Hosp Epidemiol. Jun; 1999 20(6):408-11. PubMed PMID: 10395142. [PubMed: 10395142]

25. Cosgrove SE, Qi Y, Kaye KS, Harbarth S, Karchmer AW, Carmeli Y. The impact of methicillin resistance in Staphylococcus aureus bacteremia on patient outcomes: mortality, length of stay, and hospital charges. Infect Control Hosp Epidemiol. Feb; 2005 26(2):166-74. PubMed PMID: 15756888. [PubMed: 15756888]

26. Engemann JJ, Carmeli Y, Cosgrove SE, Fowler VG, Bronstein MZ, Trivette SL, et al. Adverse clinical and economic outcomes attributable to methicillin resistance among patients with Staphylococcus aureus surgical site infection. Clin Infect Dis. Mar 1; 2003 36(5):592-8. PubMed PMID: 12594640. [PubMed: 12594640]

27. de Kraker ME, Wolkewitz M, Davey PG, Koller W, Berger J, Nagler J, et al. Clinical impact of antimicrobial resistance in European hospitals: excess mortality and length of hospital stay related to methicillin-resistant Staphylococcus aureus bloodstream infections. Antimicrobial agents and chemotherapy. Apr; 2011 55(4):1598-605. PubMed PMID: 21220533. Pubmed Central PMCID: 3067153. [PubMed: 21220533]

28. Rubin RJ, Harrington CA, Poon A, Dietrich K, Greene JA, Moiduddin A. The economic impact of Staphylococcus aureus infection in New York City hospitals. Emerging infectious diseases. JanFeb;1999 5(1):9-17. PubMed PMID: 10081667. Pubmed Central PMCID: 2627695. [PubMed: 10081667]

29. Climo MW, Sepkowitz KA, Zuccotti G, Fraser VJ, Warren DK, Perl TM, et al. The effect of daily bathing with chlorhexidine on the acquisition of methicillin-resistant Staphylococcus aureus, vancomycin-resistant Enterococcus, and healthcare-associated bloodstream infections: results of a quasi-experimental multicenter trial. Crit Care Med. Jun; 2009 37(6):1858-65. PubMed PMID: 19384220. Epub 2009/04/23. eng. [PubMed: 19384220]

30. Huang SS, Septimus E, Kleinman K, Moody J, Hickok J, Avery TR, et al. Targeted versus universal decolonization to prevent ICU infection. N Engl J Med. Jun 13; 2013 368(24):2255-65. PubMed PMID: 23718152. [PubMed: 23718152]

31. Stelfox HT, Bates DW, Redelmeier DA. Safety of patients isolated for infection control. JAMA : the journal of the American Medical Association. Oct 8; 2003 290(14):1899-905. PubMed PMID: 14532319.

32. Puzniak LA, Gillespie KN, Leet T, Kollef M, Mundy LM. A cost-benefit analysis of gown use in controlling vancomycin-resistant Enterococcus transmission: is it worth the price? Infect Control Hosp Epidemiol. May; 2004 25(5):418-24. PubMed PMID: 15188849. [PubMed: 15188849] 
33. Bureau of Labor Statistics. Occupational employment statistics: May 2012 national occupational employment and wage estimates. U.S. Bureau of Labor Statistics Division of Occupational Employment Statistics; United States Washington, D.C.: 2012. Available from: http:// www.bls.gov [2013 September 4]

34. United States Department of Health \& Human Services. HCUP facts and figures: statistics on hospital-based care in the United States. AHRQ: Agency for Healthcare Research and Quality; Rockville, MD: 2011. Available from: http://hcupnet.ahrq.gov/HCUPnet.jsp [2013 September 4]

35. Harbarth S, Hawkey PM, Tenover F, Stefani S, Pantosti A, Struelens MJ. Update on screening and clinical diagnosis of meticillin-resistant Staphylococcus aureus (MRSA). Int J Antimicrob Agents. Feb; 2011 37(2):110-7. PubMed PMID: 21163628. Epub 2010/12/18. eng. [PubMed: 21163628]

36. Tubbicke A, Hubner C, Hubner NO, Wegner C, Kramer A, Flessa S. Cost comparison of MRSA screening and management - a decision tree analysis. BMC health services research. 2012; 12:438. PubMed PMID: 23198880. Pubmed Central PMCID: 3553071. [PubMed: 23198880]

37. Malhotra-Kumar S, Abrahantes JC, Sabiiti W, Lammens C, Vercauteren G, Ieven M, et al. Evaluation of chromogenic media for detection of methicillin-resistant Staphylococcus aureus. J Clin Microbiol. Apr; 2010 48(4):1040-6. PubMed PMID: 20164268. Pubmed Central PMCID: 2849574. [PubMed: 20164268]

38. Tom TS, Kruse MW, Reichman RT. Update: Methicillin-resistant Staphylococcus aureus screening and decolonization in cardiac surgery. The Annals of thoracic surgery. Aug; 2009 88(2): 695-702. PubMed PMID: 19632455. [PubMed: 19632455]

39. Murthy A, De Angelis G, Pittet D, Schrenzel J, Uckay I, Harbarth S. Cost-effectiveness of universal MRSA screening on admission to surgery. Clin Microbiol Infect. Dec; 2010 16(12): 1747-53. PubMed PMID: 20331684. [PubMed: 20331684]

40. Kunori T, Cookson B, Roberts JA, Stone S, Kibbler C. Cost-effectiveness of different MRSA screening methods. J Hosp Infect. Jul; 2002 51(3):189-200. PubMed PMID: 12144798. [PubMed: 12144798]

41. Jernigan JA, Clemence MA, Stott GA, Titus MG, Alexander CH, Palumbo CM, et al. Control of methicillin-resistant Staphylococcus aureus at a university hospital: one decade later. Infection Control and Hospital Epidemiology. Dec; 1995 16(12):686-96. PubMed PMID: 8683086. Epub 1995/12/01. eng. [PubMed: 8683086] 


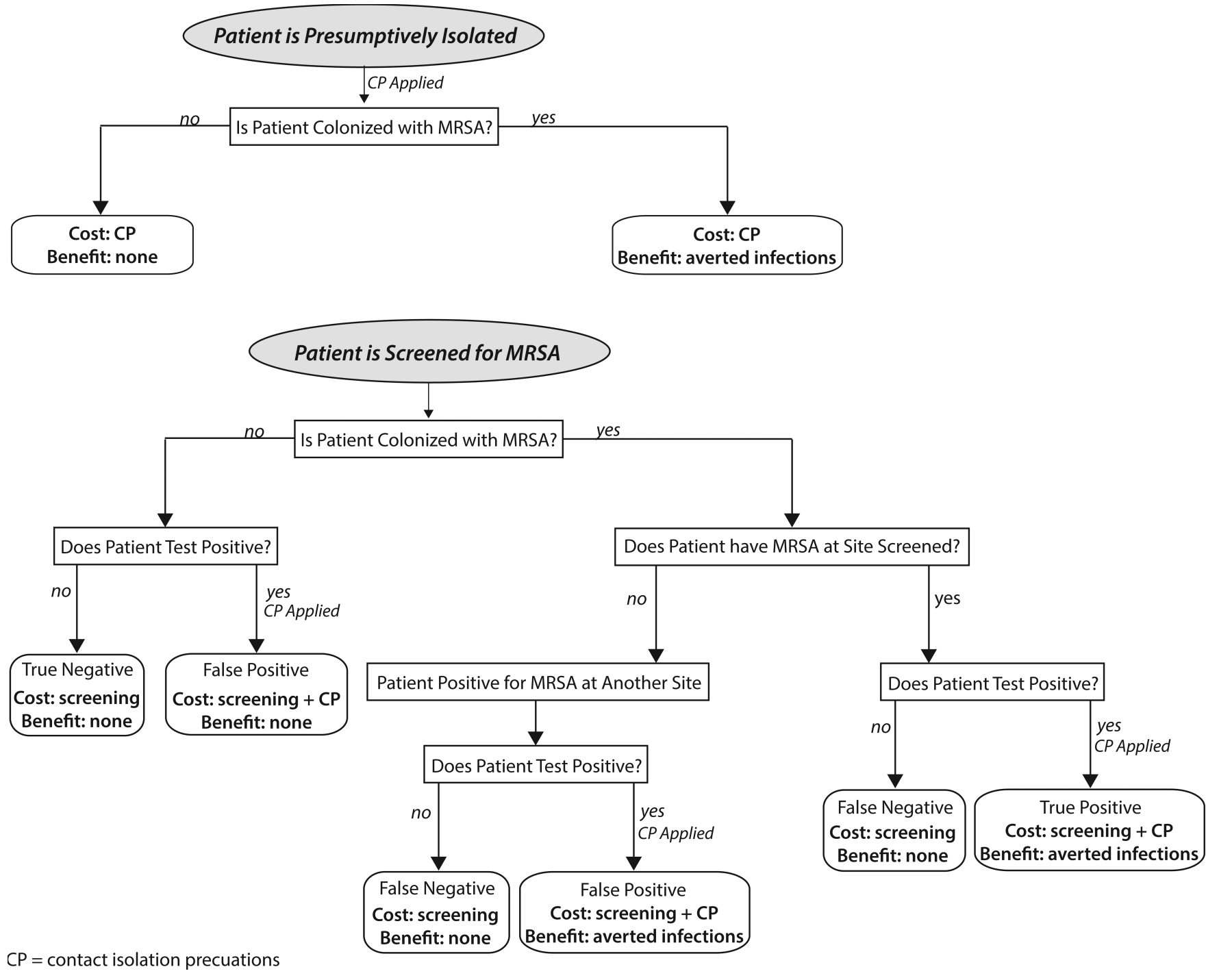

Figure 1.

Cost-Benefit model structure. Patients enter the model as MRSA colonized at each prespecified body site or not colonized. MRSA colonized patients are detected based on the sensitivity and specificity determined from the literature. Costs and benefits are allocated based on results of the screening tests and benefit of contact precautions.

$\mathrm{CP}=$ contact precautions; MRSA = Methicillin-resistant Staphylococcus aureus; "True" negative $=$ An MRSA carrier is not detected because the wrong site was chosen for surveillance, e.g. a patient colonized in the groin only who undergoes nares-only surveillance. 
A) Contact precautions averts 0.005 infections per isolated carrier

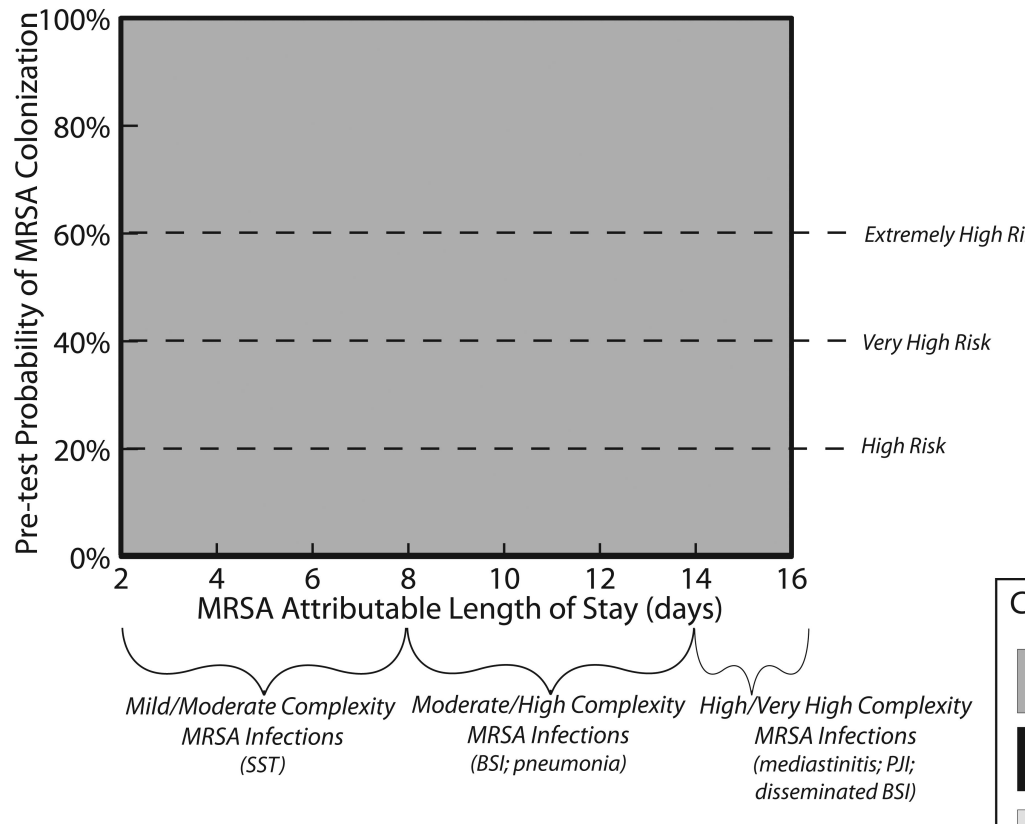

B) Contact precautions averts 0.015 infections per isolated carrier

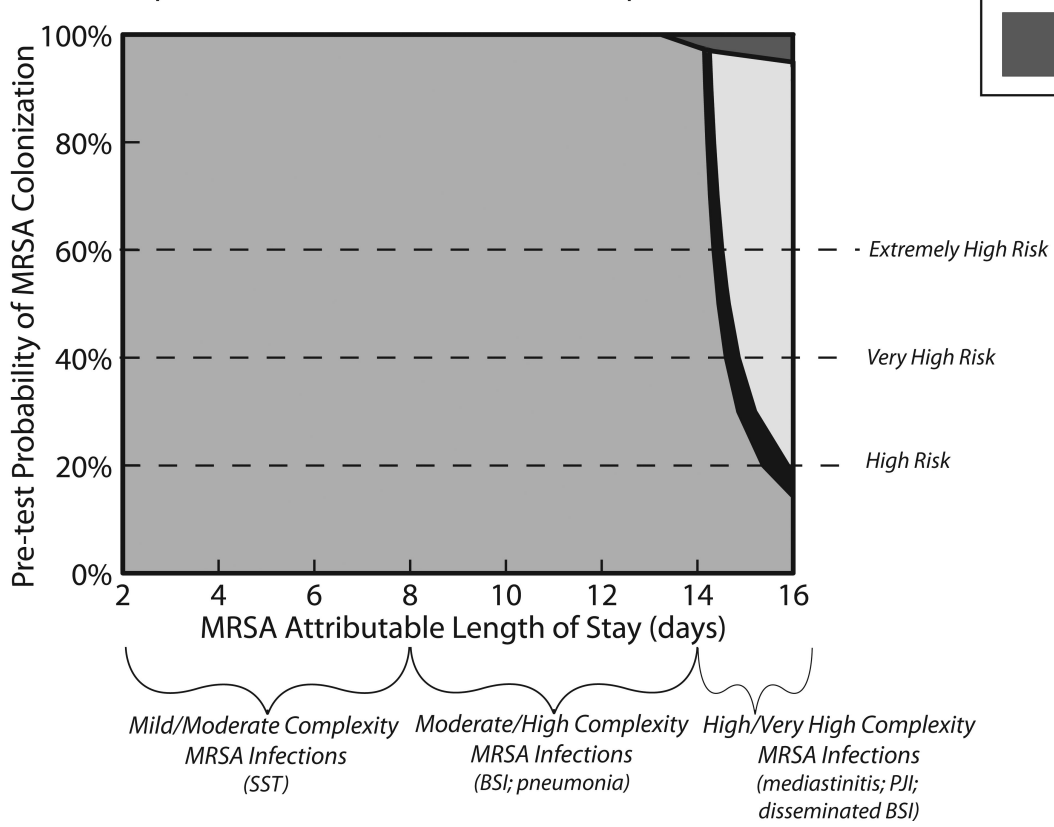

Cost Minimizing Strategy:

Nares

Nares/Oropharynx

Three Site Swab

Presumptive Isolation

Figure 2.

Sensitivity analysis of increasing the impact of MRSA infection, measured as attributable length of stay [y-axis], and efficacy of contact precautions, measured as number of infections averted [x-axis]. The shaded area represents the optimal surveillance strategy, i.e. cost=benefits or costs<benefits. The sensitivity analysis is presented for baseline conditions of MRSA colonization (10\%) and for colonization prevalence of $20 \%$ and $30 \%$.

Units of number of infections averted are per MRSA carrier placed in contact isolation. 
Table 1

Model input parameters, values, and sources.

\begin{tabular}{|c|c|c|c|c|}
\hline Parameter & Distribution & Mean or Median ${ }^{\wedge}$ & Range, Standard Deviation & Source \\
\hline \multicolumn{5}{|l|}{ Costs (2014 \$US) } \\
\hline Swab & & 1 & & (10) \\
\hline PCR Materials & & 25 & & (10) \\
\hline Chromogenic Agar Materials & & 3.5 & & (10) \\
\hline Gloves & gamma & 0.10 & 0.007 & $(10,32)$ \\
\hline Gown & & 1.01 & & (10) \\
\hline RN Hourly Wage & triangular & $33.40^{\wedge}$ & $27.37-40.14$ & (33) \\
\hline Technician Hourly Wage & triangular & $18.99^{\wedge}$ & $12.65-29.44$ & (33) \\
\hline Cost of Hospital Bed Day ${ }^{*}$ & gamma & $1,507.92$ & 205.71 & (34) \\
\hline \multicolumn{5}{|l|}{ Probabilities } \\
\hline PCR Sensitivity & beta & 0.87 & 0.075 & $(35-39)$ \\
\hline PCR Specificity & beta & 0.96 & 0.022 & $(35-39)$ \\
\hline Chromogenic Agar Sensitivity & beta & 0.82 & 0.007 & $(35-39)$ \\
\hline Chromogenic Agar Specificity & beta & 0.99 & 0.0007 & $(35-39)$ \\
\hline MRSA Prevalence on Admission & triangular & $0.095^{\wedge}$ & $0.06-0.20$ & (18) \\
\hline Carriers Detectable by Nares & triangular & $0.72^{\wedge}$ & $0.63-0.73$ & (18) \\
\hline Carriers Detectable by Orophayrnx & uniform & - & $0.15-0.20$ & $(18)$ \\
\hline \multicolumn{5}{|l|}{ Durations } \\
\hline $\begin{array}{l}\text { Hospital Length of Stay for Admitted Patients } \\
\text { (days) } * \dagger\end{array}$ & gamma & 5.0 & 0.42 & (34) \\
\hline Nurse Time Don/Doff (minutes) & & 1 & & $(10,32,40)$ \\
\hline Contacts per Day & triangular & $35^{\wedge}$ & $25-50$ & $(9,41)$ \\
\hline $\begin{array}{l}\text { Technician Time to Process PCR Sample } \\
\text { (minutes) }\end{array}$ & & 7.5 & & $(10,32,40)$ \\
\hline $\begin{array}{l}\text { Technician Time to Process Chromogenic } \\
\text { Agar Sample (minutes) }\end{array}$ & & 3.5 & & Expert Opinion \\
\hline \multicolumn{5}{|l|}{ Sensitivity Analysis } \\
\hline Parameter & & Base Case Scenario & Range of Values Tested & Sources \\
\hline $\begin{array}{l}\text { Number of MRSA Infections Averted per } \\
\text { Isolated MRSA Carrier }\end{array}$ & & 0.005 & $0.005,0.015,0.03$ & $(8-11,15,23)$ \\
\hline MRSA Attributable Length of Stay & & 6 & $2-16$ & $(24-28)$ \\
\hline MRSA Prevalence of Admitted Patients & & Distribution listed above & $0.095-0.30$ & (18) \\
\hline
\end{tabular}

Note: Carriers detectible by multi-site is the remainder of the total body colonization minus that detectable in the nares and oropharynx (this is not presented in the table)

* Values are mean and standard error

$\hat{\text { denotes median values }}$

Infect Control Hosp Epidemiol. Author manuscript; available in PMC 2016 January 01. 
Falculated from the mean cost of hospitalization divided by the mean length of stay for ICD-9 code 041.12 in HCUP for 2011 and discounted to 2014 \$US

${ }^{\dagger}$ Length of stay for all admissions in HCUP 


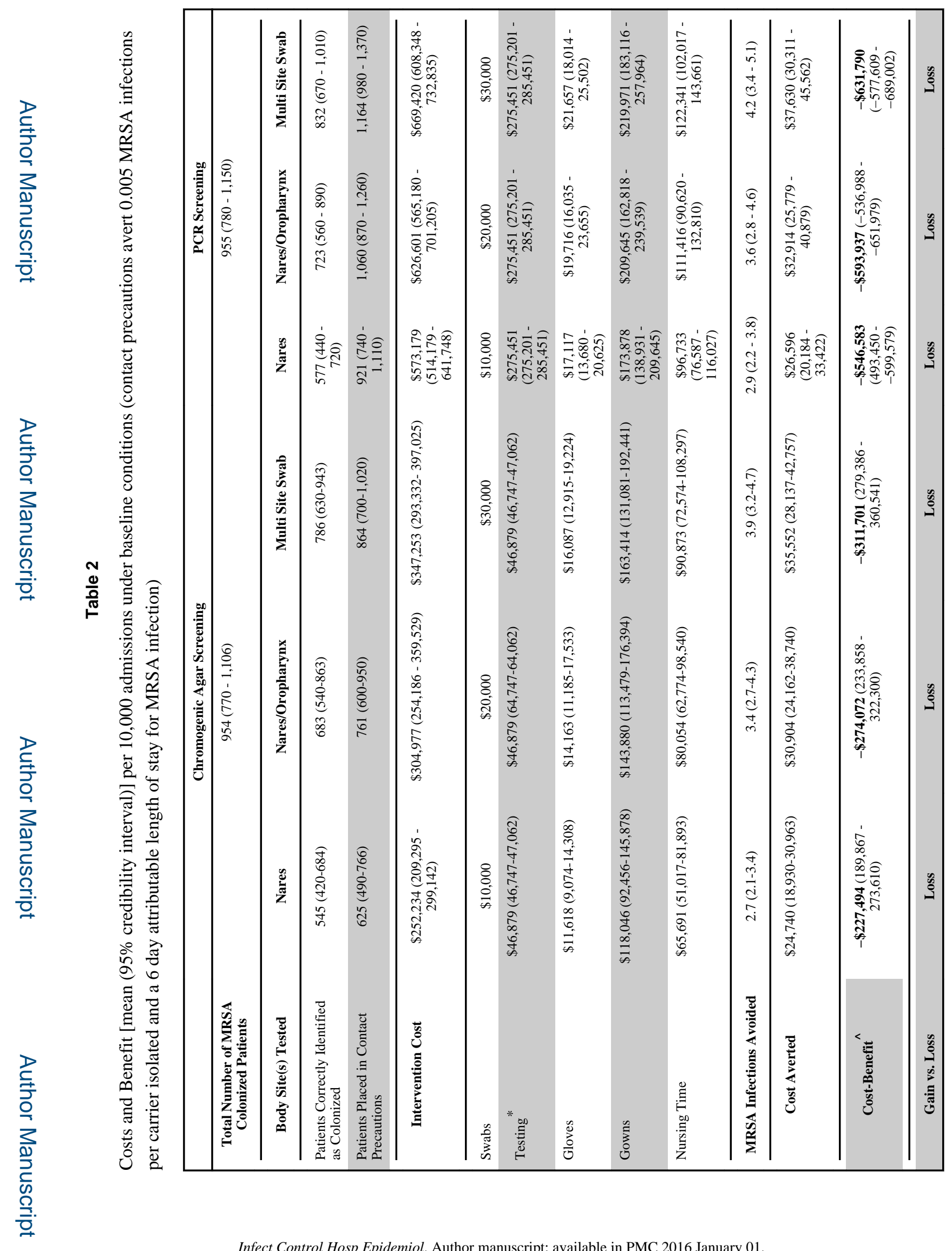



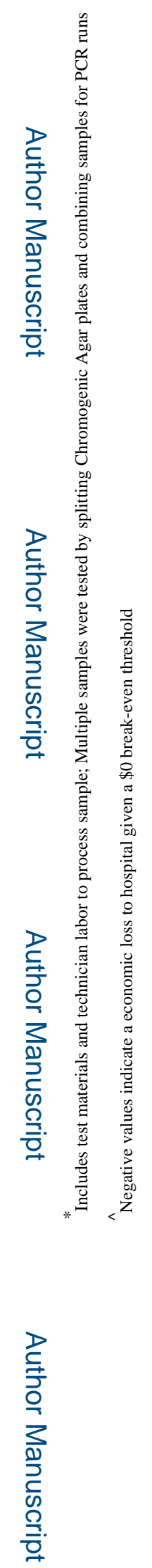

Infect Control Hosp Epidemiol. Author manuscript; available in PMC 2016 January 01. 


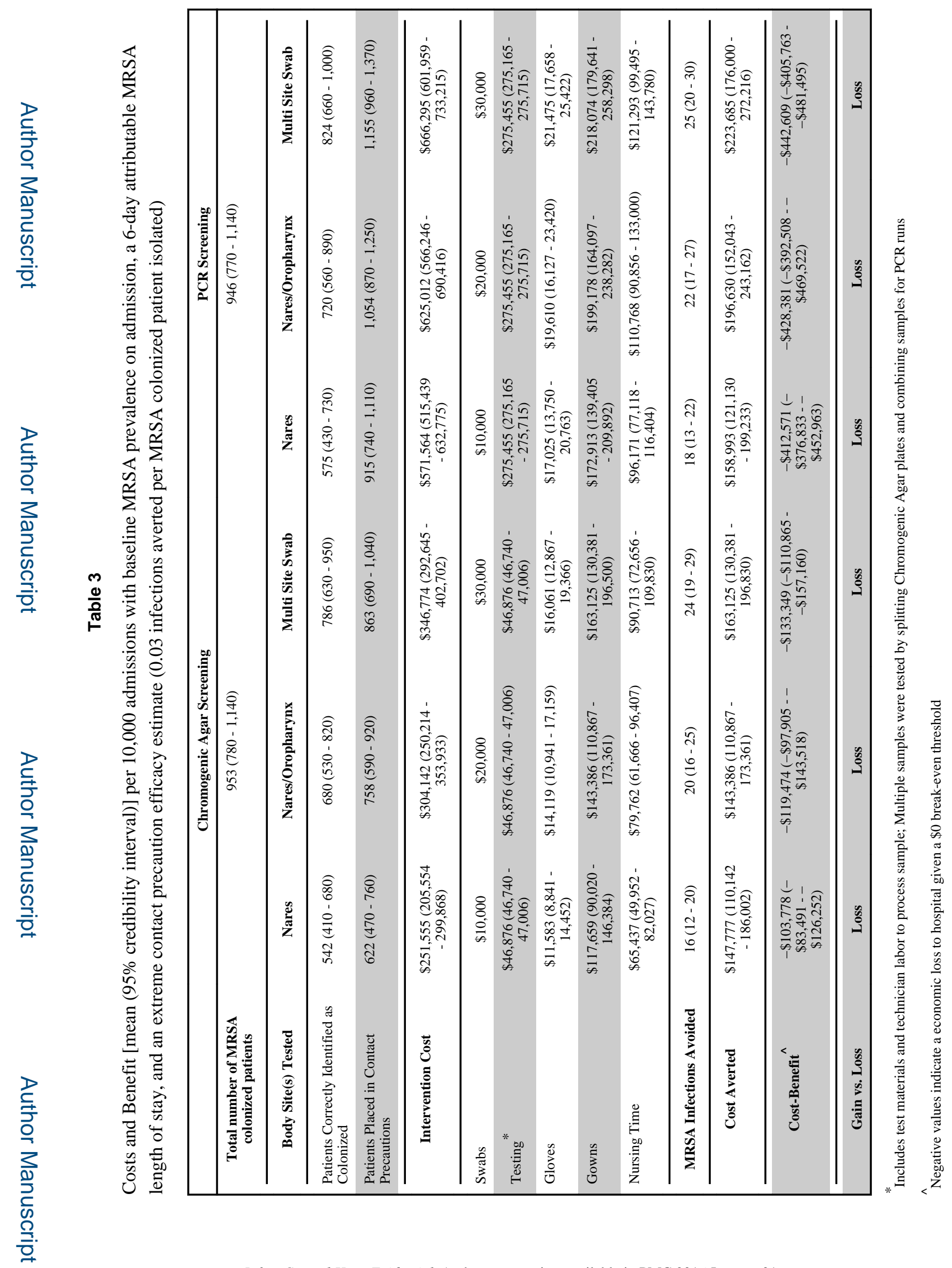

Infect Control Hosp Epidemiol. Author manuscript; available in PMC 2016 January 01. 